\title{
Vasovagal tonus index (VVTI) as an indirect assessment of remission status in canine multicentric lymphoma undergoing multi-drug chemotherapy
}

\author{
Evi Pecceu ${ }^{1} \cdot$ Brittainy Stebbing $^{1} \cdot$ Yolanda Martinez Pereira $^{1} \cdot \operatorname{Ian}$ Handel $^{1}$. \\ Geoff Culshaw $^{1} \cdot$ Hannah Hodgkiss-Geere ${ }^{1,2} \cdot$ Jessica Lawrence ${ }^{1,3}$
}

Received: 16 March 2017 / Accepted: 21 July 2017 / Published online: 8 August 2017

(C) The Author(s) 2017. This article is an open access publication

\begin{abstract}
Vasovagal tonus index (VVTI) is an indirect measure of heart rate variability and may serve as a marker of disease severity. Higher heart rate variability has predicted lower tumour burden and improved survival in humans with various tumour types. The purpose of this pilot study was to evaluate VVTI as a biomarker of remission status in canine lymphoma. The primary hypothesis was that VVTI would be increased in dogs in remission compared to dogs out of remission. Twenty-seven dogs were prospectively enrolled if they had a diagnosis of intermediate to high-grade lymphoma and underwent multidrug chemotherapy. Serial electrocardiogram
\end{abstract}

Evi Pecceu

evi.pecceu@gmail.com

Brittainy Stebbing

brittainy.stebbing@gmail.com

Yolanda Martinez Pereira

ymartin2@exseed.ed.ac.uk

Ian Handel

ian.handel@ed.ac.uk

Geoff Culshaw

Geoff.Culshaw@ed.ac.uk

Hannah Hodgkiss-Geere

H.Hodgkiss-Geere@liverpool.ac.uk

Jessica Lawrence

jlawrenc@umn.edu

1 Royal (Dick) School of Veterinary Studies \& Roslin Institute, University of Edinburgh, Roslin EH25 9RG, UK

2 Present address: Small Animal Teaching Hospital, University of Liverpool, Liverpool CH64 7TE, UK

3 Present address: College of Veterinary Medicine, University of Minnesota, St Paul, MN 55108, USA data were collected under standard conditions and relationships between VVTI, remission status and other clinical variables were evaluated. VVTI from dogs in remission (partial or complete) did not differ from dogs with fulminant lymphoma (naive or at time of relapse). Dogs in partial remission had higher VVTI than dogs in complete remission $(p=0.021)$. Higher baseline VVTI was associated with higher subsequent scores $(p<0.001)$. VVTI also correlated with anxiety level $(p=0.03)$. Based on this pilot study, VVTI did not hold any obvious promise as a useful clinical biomarker of remission status. Further investigation may better elucidate the clinical and prognostic utility of VVTI in dogs with lymphoma.

Keywords Lymphoma $\cdot$ Heart rate $\cdot$ Chemotherapy $\cdot$ Remission $\cdot$ Electrocardiogram

\section{Introduction}

Heart rate variability (HRV) is the physiologic variation in the beat-to-beat interval, or the R-R interval on an electrocardiogram (ECG) recording, which correlates with cardiac autonomic tone (Kuo et al. 2005). Variations in R-R intervals reflect vagal outflow as parasympathetic nerve traffic exerts its effects much faster than sympathetic outflow (Task Force 1996). In people, HRV is an index of cardiovascular and mortality risk in health and cardiac and renal disease (Tsuji et al. 1996; Kiviniemi et al. 2007; Oikawa et al. 2009). Several studies have investigated HRV in healthy and unhealthy dogs, with diseases including myocarditis, myxomatous mitral valve disease and diabetes mellitus, suggesting a role for VVTI as a predictive or prognostic biomarker (Calvert and Wall 2002; Doxey and Boswood 2004; Pereira et al. 2008; Manzo et al. 2009; Oliveira et al. 2012; Pirintr et al. 2012; 
Rasmussen et al. 2012; López-Alvarez et al. 2014; Martlé et al. 2014; Bogucki and Noszczyk-Nowak 2015).

The vagus nerve may be intricately involved in human cancer as part of an inflammatory reflex, in which tumourmediated inflammation triggers vagal afferent traffic to the brain to modulate immune and neuroendocrine function (Tracey 2002; Gidron et al. 2005; Mravec et al. 2006; Gidron and Ronson 2008; Golan et al. 2009; Rosas-Ballina and Tracey 2009; Irwin and Cole 2011; Olofsson et al. 2012). Recent studies have investigated the prognostic value of HRV in human cancer patients. Higher HRV was associated with longer survival time, and predicted lower tumour burden and improved survival in patients with various solid tumours (Dekker et al. 1997, Hoffmann et al. 2001, Chiang et al. 2010, Fadul et al. 2009, Giese-Davis et al. 2015, Guo et al. 2015, Kim et al. 2015). Thus HRV has potential in oncology as a biomarker of response to chemotherapy and overall prognosis (De Couck and Gidron 2013; Giese-Davis et al. 2015).

Vasovagal tonus index (VVTI) is a time domain indicator of HRV that quantifies high-frequency variations in heart rate. VVTI measurement is rapid, non-invasive and uncomplicated because it can be calculated from ECG recordings using a simple mathematical formula. Its prognostic value has been demonstrated in dogs with congestive heart failure secondary to both dilated cardiomyopathy and myxomatous mitral valve disease (Häggström et al. 1996; Doxey and Boswood 2004; Pereira et al. 2008; López-Alvarez et al. 2014). If VVTI is a reliable biomarker of disease severity, it may therefore have widespread clinical potential. Recognizing that humans with higher disease burden have significantly lower HRV compared to patients with earlier cancer stages (Entschladen et al. 2004; Gidron et al. 2005; Mantovani et al. 2008; De Couck et al. 2012; De Couck and Gidron 2013), we elected to evaluate the ability of VVTI to detect tumour burden in dogs with lymphoma. Lymphoma burden decreases rapidly in most dogs following chemotherapy, thereby permitting VVTI comparisons within each dog and across groups of dogs. The primary hypothesis of this study was that vagal tone, and therefore HRV, would increase in dogs with intermediate to high-grade lymphoma when in remission with multidrug chemotherapy.

\section{Material and methods}

\section{Dogs}

Client-owned dogs with multicentric non-Hodgkin's lymphoma were prospectively enrolled at a specialty oncology service between January 2014 and September 2015. Inclusion criteria were: stage III-V intermediate and high grade lymphoma diagnosed by cytology or histology, treatment with standardised multidrug (cyclophosphamide, hydroxydaunorubicin, vincristine, prednisone or $\mathrm{CHOP}$ ) chemotherapy and client consent

(Macdonald et al. 2005). As this was a pilot study, dogs were enrolled at any stage of treatment or remission, including at diagnosis, while undergoing chemotherapy or during routine monthly monitoring for relapse.

Exclusion criteria included evidence of behavioural aggression, systemic diseases or obvious pre-existing cardiac disease identified from a combination of clinical history, physical examination and/or echocardiography. Pre-chemotherapy echocardiography was at the discretion of the clinician, and principally performed to identify subclinical cardiac disease prior to administration of doxorubicin. The study was approved by the Institutional Veterinary Ethics and Review Committee.

\section{ECG recordings and VVTI measurements}

Standard 6-lead ECGs were recorded and printed $(50 \mathrm{~mm} / \mathrm{s})$ with a multi-channel ECG machine (Schiller AT-102 Plus ${ }^{\mathrm{TM}}$ ) collected at every chemotherapy or follow-up visit. All recordings were obtained from unsedated dogs placed in right lateral recumbency in a dimly-lit room at least $2 \mathrm{~h}$ following admission. ECG leads were attached with atraumatic clips and alcohol $\left(95 \%\right.$ ethanol, 5\% methanol; Surgical Spirit ${ }^{\mathrm{TM}}$, Vet Way Ltd., Elvington York, UK) and no electronic filters were applied. ECG trace analysis was performed manually on the first available 20 consecutive R-R intervals of sinus origin. Each dog was scored during ECG acquisition according to a 5-point rising scale of anxiety (Table 1). This anxiety score was generated by the authors using elements from anxiety scores previously published (Beata et al. 2007; Frank et al. 2006). R-R intervals were measured in millimetres and converted into milliseconds. VVTI was calculated as the natural logarithm of the variance of 20 consecutive R-R intervals as previously described (Häggström et al. 1996). This was performed by one member of the Cardiology Service (BS) who was blinded to the clinical information. Follow-up ECGs were scheduled at monthly intervals for the first 18 months, followed by every other month for 18 months.

\section{Clinical data}

Signalment was collected for each dog as well as clinical data where available, including the presence or absence of hypercalcaemia, stage, substage, immunophenotype,

Table 1 Anxiety scoring

\begin{tabular}{l}
\hline Anxiety score $0=$ no; $1=$ yes \\
\hline Attempt to rise \\
Stiff / tense \\
Panting \\
Limb withdrawal \\
Awake \\
Overall score $/ 5=$ \\
\hline
\end{tabular}


chemotherapy drugs and dosages administered, remission status, duration to remission, duration to first relapse, and development of chemotherapy-induced toxicity. Full staging was recommended for all dogs at diagnosis, however not always pursued according to clients' wishes. Minimal staging, which included lymph node measurements, haematology and biochemistry, was performed in all dogs prior to initiation of chemotherapy. Remission status was determined using standardised criteria (Vail et al. 2010). Dogs with overt lymphoma at the time of initial diagnosis or at time of relapse were documented as "out of remission (OR)" while remission status was divided as partial remission (PR) or complete clinical remission (CR). At each chemotherapy visit, lymph node measurements, physical exam and haematology was performed. Chemotherapy-induced toxicity was recorded using standardised Veterinary Cooperative Oncology Group (VCOG) criteria (VCOG 2011). At time of relapse, repeat $\mathrm{CHOP}$ was offered. For dogs that failed CHOP chemotherapy (relapsed disease during CHOP), lomustine and prednisolone was offered as first-line rescue therapy.

\section{Statistical analysis}

Statistical analyses were performed (IH) using the $\mathrm{R}$ Statistical System (R Core Team. R: A language and environment for statistical computing. R Foundation for Statistical Computing (R Core Team 2015) . Available at: https://www.R-project.org/.). VVTI scores were compared using linear models (estimated with lme function of the $\mathrm{R}$ nlme package) (Pinheiro et al. 2017). Case identifier was included as a random effect on the model intercept to reduce the impact of pseudo-replication when measurements were repeated within dogs. A first order autocorrelation term was included as VVTI measurements demonstrated autocorrelation on exploratory analysis (using the AR1 function of the R nlme package). The significance of model terms was assessed using likelihood ratio tests (LRT) on the addition of the terms. Models assessing impact of remission status used categorical remission status as a single fixed effect. Remission status was retained as a fixed effect in models assessing anxiety, substage and age. The association between baseline VVTI and later VVTI was described by Pearson product moment correlation coefficient and estimated with a random effects linear model. Predictability of remission by means of a VVTI was evaluated with a binary logistic regression model. The study involved multiple statistical tests in the exploration of hypotheses. No explicit corrections were made for this; $p$ values are presented as-is. Data are expressed as mean, median and range. A $p$-value of $<0.05$ was considered statistically significant for reporting of final variables from the models.

\section{Results}

Twenty-seven dogs with a diagnosis consistent with nonHodgkin's lymphoma were enrolled in the study. Demographics and clinical data for all dogs are shown in Table 2. Twelve dogs had echocardiography prior to doxorubicin administration. A total of 249 VVTIs were obtained, with a mean and median of 9 scores/dog and 7 scores/dog (range of 131 scores/ dog). Sixteen dogs (59\%) had a baseline VVTI at initial diagnosis (chemotherapy-naïve disease) while the remaining dogs were enrolled following initiation of chemotherapy. Of the 16 dogs with baseline VVTI scores, 14 had serial VVTI scores while in PR or CR; 2 dogs did not achieve remission (Fig. 1). Of the 11 dogs lacking baseline VVTI data prior to initiation of chemotherapy, four dogs relapsed over time, providing a VVTI when OR at least once.

No significant differences were detected between VVTIs from dogs that were OR (median 7.48, range 3.98-11.39, $n=38$ ) compared to dogs in either PR or CR (Fig. 2). Compared to CR tracings (median 7.54, range 3.90-12.33, $n=143$ ), VVTI was higher in PR tracings (median 8.12, range $4.8-11.26, n=61)$ with a mean difference of $0.59(p=0.021)$ (results with interquartile ranges in Table 1). Seven scores were obtained from dogs with stable disease (SD) or progressive disease (PD) and these scores were not included in analysis.

Dogs with higher baseline scores were more likely to have higher subsequent scores when in partial or complete remission (Fig. 1); for every unit increase in baseline VVTI, there was an average 0.62 increase in VVTI $(p<0.001)$. VVTI was associated with anxiety level, with decreasing VVTI as anxiety increased $(p=0.03)$. VVTI did not differ between dogs with substage $\mathrm{a}$ and $\mathrm{b}$ lymphoma $(p=0.28)$. Likewise, VVTI did not correlate with sex, age, lymphoma stage, hypercalcemia, chemotherapy-induced adverse effects, chemotherapy status (receiving or not receiving concurrent chemotherapy, number of treatments, number of doxorubicin treatments), breed or brachycephalic phenotype.

\section{Discussion}

This is the first study to investigate the use of VVTI in canine cancer. Humans with more advanced cancer, have been shown to have significantly lower HRV compared to patients with earlier cancer stages (Entschladen et al. 2004; Gidron et al. 2005; Mantovani et al. 2008; De Couck et al. 2012; De Couck and Gidron 2013). As disease burden in canine lymphoma decreases rapidly following the initiation of chemotherapy, canine lymphoma was a reasonable initial model disease in which to evaluate VVTI, thereby permitting comparisons within each dog and across groups of dogs.

While VVTI was significantly higher for dogs in PR compared to in CR, VVTI in either of these stages did 
Table 2 Summary of clinical data from 27 dogs with lymphoma in which vasovagal tonus index (VVTI) was measured

\begin{tabular}{|c|c|c|}
\hline \multicolumn{3}{|c|}{ Clinical Characteristics $(N=27 \mathrm{dogs})$} \\
\hline Age (years) & Median (Range) & $8(2.25-11.91)$ \\
\hline \multirow[t]{2}{*}{ Sex } & M & $18(67 \%)$ \\
\hline & $\mathrm{F}$ & $9(33 \%)$ \\
\hline Body weight (kg) & Median (Range) & $26.6(6.3-75.5)$ \\
\hline \multirow[t]{7}{*}{ Breed } & Labrador & $4(15 \%)$ \\
\hline & Border Collie & $3(11 \%)$ \\
\hline & Boxer & $3(11 \%)$ \\
\hline & Rottweiler & $2(7.5 \%)$ \\
\hline & WHWT & $2(7.5 \%)$ \\
\hline & Cross & $3(11 \%)$ \\
\hline & Other $(<2)$ & $10(37 \%)$ \\
\hline Breed type & Brachycephalic & $3(11 \%)$ \\
\hline \multirow[t]{3}{*}{ Stage } & Stage III & $9(33 \%)$ \\
\hline & Stage IV & $15(56 \%)$ \\
\hline & Stage V & $3(11 \%)$ \\
\hline \multirow[t]{2}{*}{ Substage } & Substage a & $19(70 \%)$ \\
\hline & Substage b & $8(30 \%)$ \\
\hline Hypercalcemia & & $5(19 \%)$ \\
\hline \multirow[t]{2}{*}{ Immunophenotype } & B-cell & $4(80 \%$ of samples tested $)$ \\
\hline & T-cell & $1(20 \%)$ \\
\hline \multirow[t]{3}{*}{ VVTI total scores } & 242 & $38 \mathrm{OR}$ \\
\hline & & $61 \mathrm{PR}$ \\
\hline & & $143 \mathrm{CR}$ \\
\hline \multirow[t]{3}{*}{ VVTI measures } & OR & (mean 7.77 median 7.48 IQ 6.58-9.08) \\
\hline & PR & (mean 8.00 median 8.12 IQ 6.81-8.93) \\
\hline & CR & (mean 7.54 median 7.54 IQ $6.55-8.33$ ) \\
\hline
\end{tabular}

WHWT West Highland White Terrier, $O R$ out of remission, $P R$ partial remission, $C R$ complete remission, $I Q$ Interquartile range not differ from OR. Therefore the null hypothesis was correct. The results were unexpected, as theoretically, decreased lymphoma burden in dogs in CR compared to tumour burden in PR would lead to decreased sympathetic tone, increased parasympathetic tone and thus increased VVTI. Similarly, it was expected that dogs in either CR or PR would have increased VVTI compared to dogs that were OR. Although it is unclear how HRV directly correlates to human cancer, a bi-directional relationship has been hypothesised. Higher disease burden was thought to decrease heart rate variability due to inflammation, oxidative stress and sympathetic activation. Vagal tone however is not only a consequence of tumour burden but it is also suspected to actively modulate tumour growth through anti-inflammatory effects via two separate ways. Via afferent vagal nerve fibres, the hypothalamic-pituitary-adrenal axis can be activated, leading to the production of corticosteroids and an anti-inflammatory response. Via efferent fibres, anti-inflammatory signals can be transmitted via acetylcholine to activate receptors on tissue macrophages and suppress cytokine synthesis (Gidron et al. 2005;
Mravec et al. 2006). Prior studies have shown that there is a worse prognosis in cancer when there is not an intact vagus; moreover, a causal relationship was shown between vagal nerve activation and reduced tumour volume (Erin et al. 2004; Erin et al. 2008; Erin et al. 2012). This bidirectional relationship between cancer burden and heart rate variability hampers a simplistic interpretation of higher tumour burden that results in a lower HRV and thus may offer an explanation for the unexpected results.

Dogs with higher baseline values were found to have higher subsequent VVTI scores when in remission. This suggests that there may be baseline variation across dogs regardless of intra-dog score variability. Previously a range of scores with marked variability across breeds of dogs has been published (Doxey and Boswood 2004). However, in this study, it is not the absolute value of VVTI that is important but rather the change from baseline. Brachycephalic dogs have higher vagal tone and higher VVTI, and while brachcephaly may have affected the degree to which VVTI changed from baseline, only three dogs were brachycephalic, making it unlikely that this significantly impacted the chance to detect a 
Fig. 1 Vasovagal tonus index (VVTI) values per dog in different remission statuses for dogs with available baseline scores at time of initial diagnosis (out of remission $=\mathrm{OR}$ ) and subsequent partial remission (PR) and/or complete remission $(\mathrm{CR})$ values $(N=14)$. Baseline VVTI is represented by a +

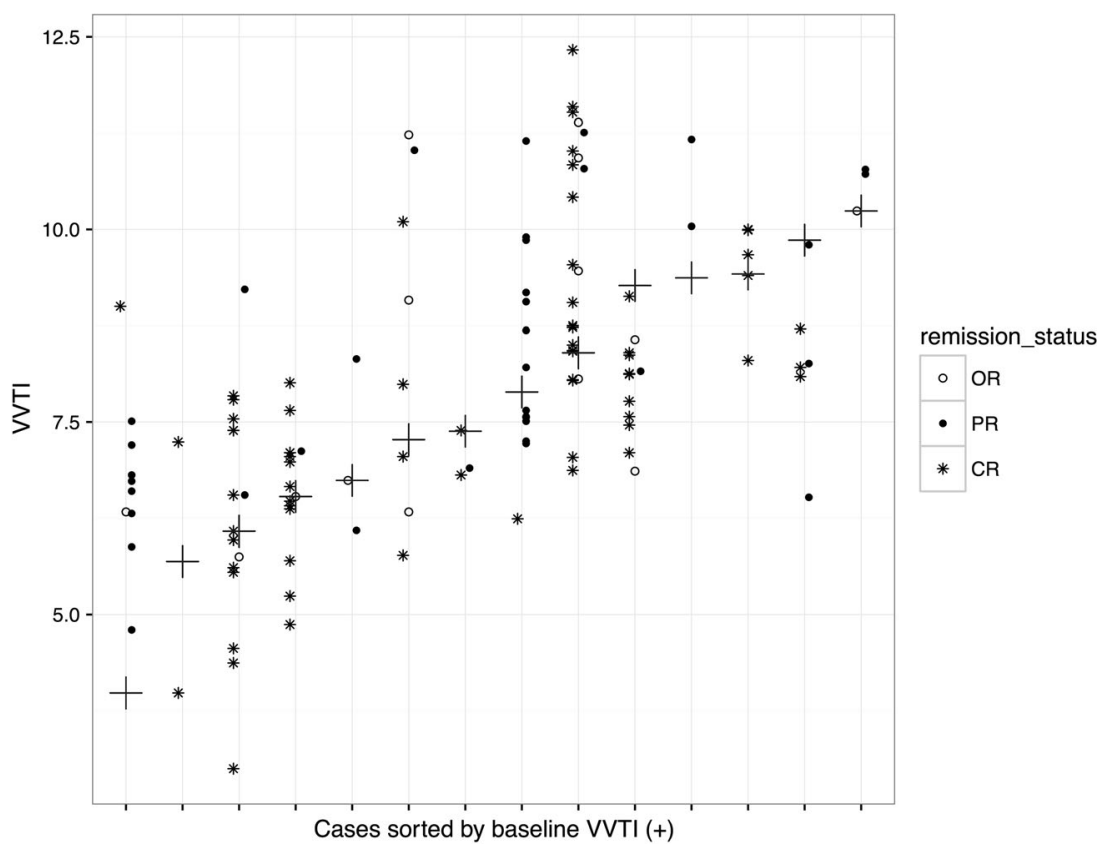

correlation between VVTI scores and remission status. Statistical analysis did not show any significant correlation between VVTI score and brachycephalic phenotype.
Higher anxiety scores were associated with lower VVTI. The protocol to obtain ECG was designed to minimise anxiety during collection of data, however some

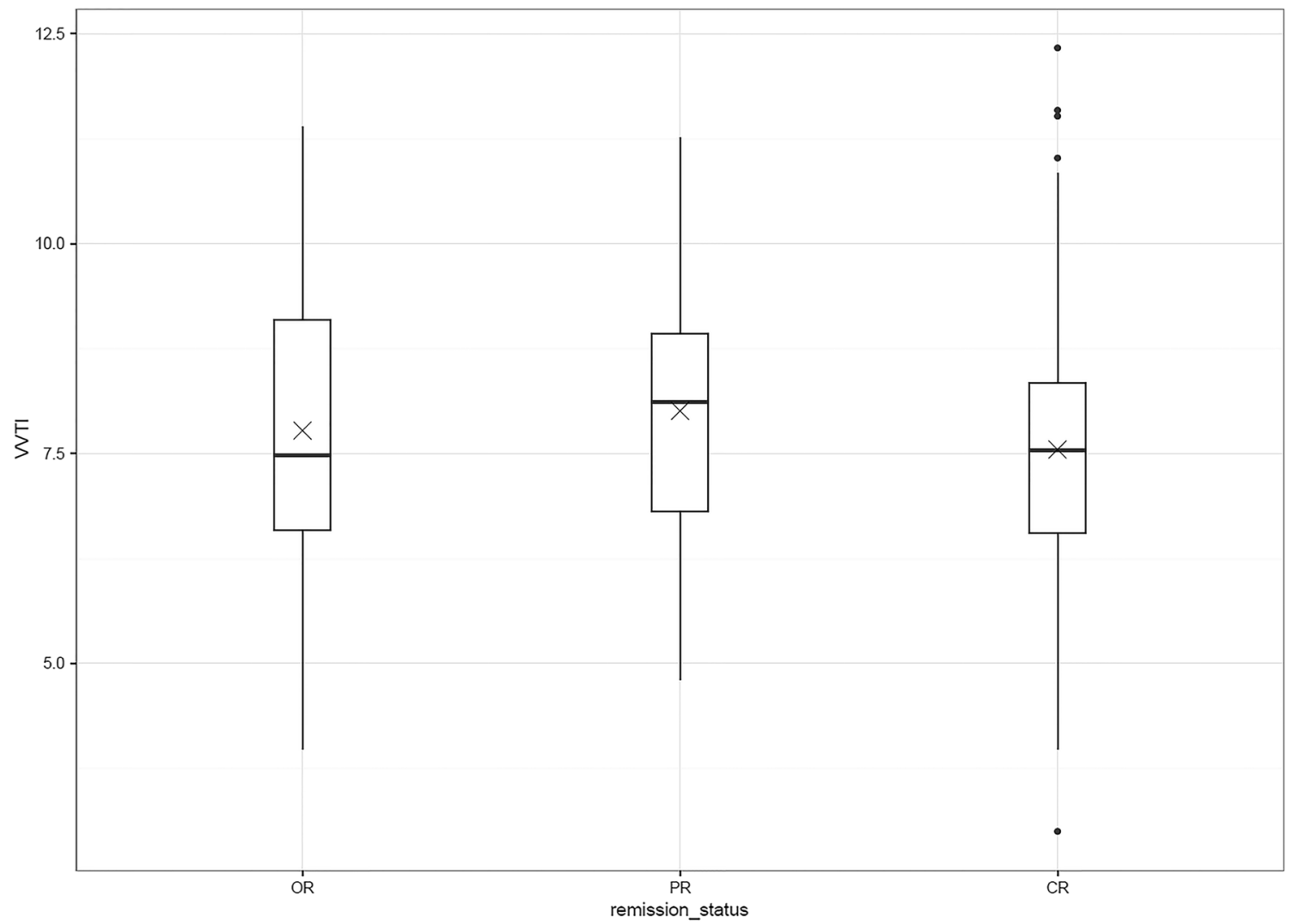

Fig. 2 Box and Whisker plot demonstrating the distribution of vasovagal tonus index (VVTI) values across remission status for the 27 dogs enrolled; horizontal line represents the median, the $\mathrm{X}$ represents the mean of scores. VVTI was significantly higher in dogs in partial remission (PR) compared to dogs in complete remission (CR). VVTI from dogs with fulminant lymphoma either at initial diagnosis or at time of relapse (out of remission $=$ OR) were not significantly different from dogs in partial and/or complete remission 
dogs still demonstrated signs of anxiety and further modifications such as prolonged acclimatisation or permitting owner presence in future studies may further reduce anxiety. As the change in VVTI from baseline was important in this study and anxiety scores did not increase in each dog over time the fact that VVTI scores from dogs in PR was higher than dogs in CR suggests the level of anxiety did not significantly influence our findings.

Dogs in which concurrent cardiac disease was suspected were not included. Echocardiography was not performed in all dogs prior to the first dose of doxorubicin. It is possible that dogs with subclinical cardiac disease were included in this study. While inclusion criteria could have required an echocardiogram prior to treatment, published literature in dogs with mitral valve disease suggests that subclinical cardiac disease would not have significantly affected VVTI (Häggström et al. 1996). Moreover, echocardiogram cannot predict doxorubicin-induced cardiotoxicity in dogs, calling into question its necessity prior to treatment (Ratterree et al. 2012; Tater et al. 2012).

Fewer values were obtained of dogs with fulminant lymphoma compared to dogs in PR or CR for multiple reasons. As canine non-Hodgkin's lymphoma is rapidly proliferative, rapid remission is achieved in most dogs within one to three weeks of starting chemotherapy. Therefore, dogs contributed more scores when in PR or CR compared to when OR. This study was designed to include dogs already receiving chemotherapy and therefore without baseline values. This was deliberate in order to maximize the number of dogs recruited to the study and to increase the number of samples for analysis. It also ensured that the chemotherapy was initiated at time of diagnosis without delay in treatment to obtain baseline VVTI scores, as sedation was often used for diagnostics.

The impact of chemotherapy on VVTI is unclear although in human oncology, reduced HRV was found following treatment with anthracyclines (Tjeerdsma et al. 1999). Doxorubicin treatments as part of the CHOP protocol in our study may have influenced the VVTI scores in this population, however exploratory analysis did not show any obvious correlation of VVTI with increasing numbers of doxorubicin doses or chemotherapy treatments overall. Additionally, VVTI values from dogs on chemotherapy were not markedly different from dogs off chemotherapy following completion of their protocol. It was not the primary aim of this pilot study to accurately evaluate associations between VVTI and clinical variables other than remission status, however it was interesting to evaluate for hypothesis-generation purposes. Likewise, as the study involved multiple statistical comparisons there was a risk of increased type I errors. There were no clear associations with multiple clinical variables and VVTI, and although it is possible that a larger study could reveal an association, VVTI may instead be an independent variable.

\section{Conclusions}

In conclusion, there were no significant differences in VVTI between dogs in remission compared to dogs with overt lymphoma in this initial pilot study. Surprisingly, PR VVTIs were higher than VVTIs of dogs in CR. This is the first study to indicate that VVTI varies over the course of canine lymphoma treatment. Further studies will be essential to improve understanding of the pathophysiology of autonomic tone in dogs with lymphoma or other neoplastic diseases. A larger study population will be required to determine if clear prognostic potential exists, as has been shown in humans.

\section{Compliance with ethical standards}

Conflict of interest The authors declare that they have no conflict of interest.

Open Access This article is distributed under the terms of the Creative Commons Attribution 4.0 International License (http:// creativecommons.org/licenses/by/4.0/), which permits unrestricted use, distribution, and reproduction in any medium, provided you give appropriate credit to the original author(s) and the source, provide a link to the Creative Commons license, and indicate if changes were made.

\section{References}

Beata C, Beaumont-Graff E, Diaz C, Marion M, Massal N, Marlois N, Muller G, Lefranc C (2007) Effects of alpha-casozepine (Zylkene) versus selegiline hydrochloride (Selgian, Anipryl) on anxiety disorders in dogs. J Vet Behav 2:175-183

Bogucki S, Noszczyk-Nowak A (2015) Short-term heart rate variability (HRV) in healthy dogs. Pol J Vet Sci 18:307-312. doi:10.1515/pjvs2015-0040

Calvert CA, Wall M (2002) Evaluation of stability over time for measures of heart-rate variability in overtly healthy Doberman pinschers. Am J Vet Res 63:53-59

Chiang JK, Koo M, Kuo TB, Fu TB (2010) Association between cardiovascular autonomic functions and time to death in patients with terminal hepatocellular carcinoma. J Pain Symptom Manag 39: 673-679. doi:10.1016/j.jpainsymman.2009.09.014

De Couck M, Gidron Y (2013) Norms of vagal nerve activity, indexed by heart rate variability, in cancer patients. Cancer Epidemiol 37:737741. doi:10.1016/j.canep.2013.04.016

De Couck M, Mravec B, Gidron Y (2012) You may need the vagus nerve to understand pathophysiology and to treat diseases. Clin Sci (Lond) 122:323-328. doi:10.1042/CS20110299

Dekker JM, Schouten EG, Klootwijk P, Pool J, Swenne CA, Kromhout D (1997) Heart rate variability from short electrocardiographic recordings predicts mortality from all causes in middle-aged and elderly men. The Zutphen Study. Am J Epidemiol 145:899-908

Doxey S, Boswood A (2004) Differences between breeds of dog in a measure of heart rate variability. Vet Rec 4:713-717 
Entschladen F, Drell TL, Lang K, Joseph J, Zaenker KS (2004) Tumour-cell migration, invasion, and metastasis: navigation by neurotransmitters. Lancet Oncol 5:254-258. doi:10.1016/ S1470-2045(04)01431-7

Erin N, Boyer PJ, Bonneau RH, Clawson GA, Welch DR (2004) Capsaicin-mediated denervation of sensory neurons promotes mammary tumor metastasis to lung and heart. Anticancer Res 24:10031009. doi:10.1016/j.regpep.2012.08.001

Erin N, Akdas Barkan G, Harms JF, Clawson GA (2008) Vagotomy enhances experimental metastases of 4THMpc breast cancer cells and alters substance P level. Regul Pept 151:35-42. doi:10.1016/j. regpep.2008.03.012

Erin N, Duymuş O, Oztürk S, Demir N (2012) Activation of vagus nerve by semapimod alters substance $\mathrm{P}$ levels and decreases breast cancer metastasis. Regul Pept 179:101-108. doi:10. 1016/j.regpep.2012.08.001

Fadul N, Strasser F, Palmer JL, Yusuf SW, Guo Y, Li Z, Allo J, Bruera E (2009) The association between autonomic dysfunction and survival in male patients with advanced cancer: a preliminary report. J Pain Symptom Manag 39:283-290. doi: 10.1016/j.jpainsymman.2009.06.014

Frank D, Gauthier A, Bergeron R (2006) Placebo-controlled double-blind clomipramine trial for the treatment of anxiety or fear in beagles during ground transport. Can Vet J 47:1102-1108. doi:10.1016/j. jveb.2007.02.002

Gidron Y, Ronson A (2008) Psychosocial factors, biological mediators, and cancer prognosis: a new look at an old story. Curr Opin Oncol 20:386-392. doi:10.1097/CCO.0b013e3282fbcd0d

Gidron Y, Perry H, Glennie M (2005) Does the vagus nerve inform the brain about preclinical tumours and modulate them? Lancet Oncol 6:245-248. doi:10.1016/S1470-2045(05)70096-6

Giese-Davis J, Wilhelm FH, Tamagawa R, Palesh O, Neri E, Taylor CB, Kraemer HC, Spiegel D (2015) Higher vagal activity as related to survival in patients with advanced breast cancer: an analysis of autonomic dysregulation. Psychosom Med 77:346-355. doi:10.1097/ PSY.0000000000000167

Golan H, Kennedy JA, Frenkel A, Parmet Y, Feintuch A, Levi O, Gidron Y (2009) Brain mapping of patients with lung cancer and controls: inquiry into tumor-to-brain communication. J Nucl Med 50:10721075. doi:10.2967/jnumed.108.061085

Guo Y, Koshy S, Hui D, Palmer JL, Shin K, Bozkurt M, Yusuf SW (2015) Prognostic value of heart rate variability in patients with cancer. J Clin Neurophysiol 32:516-520.doi:10.1097/WNP. 0000000000000210

Häggström J, Hamlin RL, Hansson K, Kvart C (1996) Heart rate variability in relation to severity of mitral regurgitation in cavalier king Charles spaniels. J Small Anim Pract 37:69-75

Hoffmann J, Grimm W, Menz V, Wied M, Sprenger A, Arnold R, Maisch B (2001) Prognostic value of heart rate variability analysis in patients with carcinoid syndrome. Digestion 63:35-42

Irwin M, Cole S (2011) Reciprocal regulation of the neural and innate immune systems. Nat Rev Immunol 11:625-632. doi: $10.1038 /$ nri3042

Kim K, Chae J, Lee S (2015) The role of heart rate variability in advanced non-small-cell lung cancer patients. J Palliat Care 31:103-108

Kiviniemi AM, Tulppo MP, Wichterle D, Hautala AJ, Tiinanen S, Seppänen T, Mäkikallio TH, Huikuri HV (2007) Novel spectral indexes of heart rate variability as predictors of sudden and nonsudden cardiac death after an acute myocardial infarction. Ann Med 39:54-62. doi:10.1080/07853890600990375

Kuo TB, Lai CJ, Huang YT, Yang CC (2005) Regression analysis between heart rate variability and baroreflex-related vagus nerve activity in rats. J Cardiovasc Electrophysiol 16:864-869. doi:10.1111/j. 1540-8167.2005.40656.x

López-Alvarez J, Boswood A, Moonarmart W, Hezzell MJ, Lotter N, Elliott J (2014) Longitudinal electrocardiographic evaluation of dogs with degenerative mitral valve disease. J Vet Intern Med 28: 393-400. doi:10.1111/jvim.12311

MacDonald VS, Thamm DH, Kurzman ID, Turek MM, Vail DM (2005) Does L-asparaginase influence efficacy or toxicity when added to a standard CHOP protocol for dogs with lymphoma? J Vet Intern Med 19:732-736

Mantovani A, Allavena P, Sica A, Balkwill F (2008) Cancer-related inflammation. Nature 454:436-444. doi:10.1038/nature07205

Manzo A, Ootaki Y, Ootaki C, Kamohara K, Fukamachi K (2009) Comparative study of heart rate variability between healthy human subjects and healthy dogs, rabbits and calves. Lab Anim 43:41-45. doi:10.1258/la.2007.007085

Martlé V, Bavegems V, Van Ham L, Boon P, Vonck K, Raedt R, Sys S, Bhatti S (2014) Evaluation of heart rate variability in dogs during standard and microburst vagus nerve stimulation: a pilot study. Veterinary journal (London, England: 1997) 202:651-653. doi: 10. 1016/j.tvj1.2014.09.009

Mravec B, Gidron Y, Kukanova B, Bizik J, Kiss A, Hulin I (2006) Neural-endocrine-immune complex in the central modulation of tumorigenesis: facts, assumptions, and hypotheses. J Neuroimmunol 180:104-116. doi:10.1016/j.jneuroim.2006.07.003

Oikawa K, Ishihara R, Maeda T, Yamaguchi K, Koike A, Kawaguchi H, Tabata Y, Murotani N, Itoh H (2009) Prognostic value of heart rate variability in patients with renal failure on hemodialysis. Int J Cardiol 131:370-377. doi:10. 1016/j.ijcard.2007.10.033

Oliveira MS, Muzzi RA, Araújo RB, Muzzi LA, Ferreira DF, Nogueira R, Silva EF (2012) Heart rate variability parameters of myxomatous mitral valve disease in dogs with and without heart failure obtained using 24-hour Holter electrocardiography. Vet Rec 170:622. doi:10. 1136/vr.100202

Olofsson P, Rosas-Ballina M, Levine YA, Tracey KJ (2012) Rethinking inflammation: neural circuits in the regulation of immunity. Immunol Rev 248:188-204. doi:10.1111/j.1600-065X.2012. 01138.x

Pereira YM, Woolley R, Culshaw G, French A, Martin M (2008) The vasovagal tonus index as a prognostic indicator in dogs with dilated cardiomyopathy. J Small Anim Pract 49:587-592. doi:10.1111/j. 1748-5827.2008.00654.x

Pinheiro J, Bates D, DebRoy S, Sarkar D, R Core Team (2017)_Nlme: linear and nonlinear mixed effects models. $\mathrm{R}$ package version 3.1131. https://CRAN.R-project.org/package $=$ nlme

Pirintr P, Chansaisakorn W, Trisiriroj M, Kalandakanond-Thongsong SW, Buranakarl C (2012) Heart rate variability and plasma norepinephrine concentration in diabetic dogs at rest. Vet Res Commun 36: 207-214. doi:10.1007/s11259-012-9531-0

Rasmussen CE, Falk T, Zois NE, Moesgaard SG, Häggström J, Pedersen HD, Ablad B, Nilsen HY, Olsen LH (2012) Heart rate, heart rate variability, and arrhythmias in dogs with myxomatous mitral valve disease. J Vet Intern Med 26:76-84. doi: 10.1111/j.1939-1676.2011.00842.x

Ratterree W, Gieger T, Pariaut R, Saelinger C, Strickland K (2012) Value of echocardiography and electrocardiography as screening tools prior to doxorubicin administration. J Am Anim Hosp Assoc 48:8996. doi:10.5326/JAAHA-MS-5680

R Core Team (2015) R: A language and environment for statistical computing. R Foundation for Statistical Computing, Vienna, Austria. URL https://www.R-project.org/

Rosas-Ballina M, Tracey KJ (2009) Cholinergic control of inflammation. J Intern Med 265:663-679. doi:10.1111/j.1365-2796. 2009.02098.x

Task Force of the European Society of Cardiology and the North American Society Pacing and Electrophysiology (1996) Heart rate variability: standards of measurement, physiological interpretation and clinical use. Circulation 93:1043-1065 
Tater G, Eberle N, Hungerbuehler S, Joetzke A, Nolte I, Wess G, Betz D (2012) Ventricular fractional shortening in 108 dogs with malignant lymphoma undergoing chemotherapy with a cyclic combination protocol including doxorubicin. Tierarztl Prax Ausg K Kleintiere Heimtiere 40(4):261-266

Tjeerdsma G, Meinardi MT, van Der Graaf WT, van Den Berg MP, Mulder NH, Crijns HJ, de Vries EG, van Veldhuisen DJ (1999) Early detection of anthracycline induced cardiotoxicity in asymptomatic patients with normal left ventricular systolic function: autonomic versus echocardiographic variables. Heart $81: 419-423$

Tracey K (2002) The inflammatory reflex. Nature 420:853-859. doi:10. 1038/nature 01321
Tsuji H, Larson MG, Venditti FJ Jr, Manders ES, Evans JC, Feldman CL, Levy D (1996) Impact of reduced heart rate variability on risk for cardiac events. The Framingham Heart Study. Circulation 94:2850-2855

Vail DM, Michels GM, Khanna C, Selting KA, London CA, Veterinary Cooperative Oncology Group (2010) Response evaluation criteria for peripheral nodal lymphoma in dogs (v1.0) - a veterinary cooperative oncology group (VCOG) consensus document. Vet Comp Oncol 8:28-37. doi:10.1111/j.1476-5829.2009.00200.x

Veterinary Cooperative Oncology Group (2011) Common terminology criteria for adverse events (VCOG-CTCAE) following chemotherapy or biological antineoplastic therapy in dogs and cats v1.1. Vet Comp Oncol. doi:10.1111/j.1476-5829.2011.00283.x 